\title{
Colonisation of the sheep rumen with polycentric anaerobic fungi isolated from cattle
}

\author{
MW Phillips, GLR Gordon \\ CSIRO Animal Production, Locked Bag 1, Blacktown, New South Wales 2148, Australia
}

Anaerobic fungi are known to be normal inhabitants of the rumen and hindgut of large herbivores. Recent evidence suggests that these organisms play an important role in the digestion of diets high in fibre (Gordon and Phillips 1993, Lett Appl Microbiol, 17, 220-223). The different genera of fungi are characterised by having predominantly monocentric or polycentric modes of growth (Barr et al, 1989, Canad J Bot, 67, 2815-2824). Although monocentric fungi are ubiquitous colonisers of the sheep rumen, polycentric fungi have not been found in Australian sheep. In contrast both types of fungi can be found in the cattle rumen. The aim of this study was to test whether polycentric fungi were able to colonise the rumen of sheep after indigenous populations of anaerobic fungi had been removed by antibiotics.

Polycentric fungi (all Orpinomyces spp) were isolated from the rumen digesta of cattle using a roll tube method (Phillips and Gordon, 1989, Appl Environ Micobiol, 55, 1695-1702) and subsequently maintained as pure cultures on agar plate media. Sheep (Merino $x$ BorderLeicester cross) with permanent rumen fistulae were kept in metabolism crates in the same room but without direct contact between individuals and given ad libitum a diet of $75 \%$ chopped barley straw and $25 \%$ chopped lucerne hay ( 8 meals/day). Indigenous populations of anaerobic fungi were removed by treating the animals with a combination of tetronasin and cycloheximide (Gordon and Phillips, 1993). After antibiotic treatment had ceased the rumens of four sheep were inoculated on three consecutive days with 100 $\mathrm{ml}$ of the selected fungal isolates grown in $1 \%$ straw broths. Each sheep received a different mixture of pure cultures of polycentric fungi ( 12 isolates, 3 each per sheep). The numbers of fungi present in the rumen of the test sheep were assessed using roll tube counts on strained digesta (Phillips and Gordon, 1989). Polycentric fungi were easily differentiated from indigenous monocentric fungi by the nature of their rapidly spreading colonies.

Polycentric fungi (PF) colonised each of the inoculated sheep. The relatively low numbers of fungal colonies cultured from sheep rumen digesta were similar to the numbers found in cattle rumen digesta (unpublished results) and may represent low zoospore production by polycentric fungi (Phillips, 1989, Roles of Protozoa and Fungi in Ruminant Digestion, RA Leng et al eds, 247-249). Polycentric fungi were the only fungi present for the duration of the experiment in two sheep while in the other sheep indigenous monocentric fungi (MF) recolonised. Although there was a drop in the numbers of PF when MF were present, the two types of fungi were able to coexist ; PF were still present seven weeks after inoculation (data not shown). Subsequent experiments have shown similar results when sheep were inoculated with individual pure cultures of these PF. The ability of non-indigenous fungi to colonise and persist in the sheep rumen even when indigenous fungi were present indicates that improving fibre digestion by intra-ruminal inoculation of highly fibrolytic non-indigenous fungi may be possible.

\begin{tabular}{|c|c|c|c|c|c|c|c|c|}
\hline \multirow[b]{3}{*}{ Days } & & & \multicolumn{4}{|c|}{ Number of fungal colonies/ml } & & \\
\hline & \multicolumn{2}{|c|}{ sheep 34} & \multicolumn{2}{|c|}{ sheep 35} & \multicolumn{2}{|c|}{ sheep 32} & \multicolumn{2}{|c|}{ sheep 39} \\
\hline & MF & PF & MF & PF & MF & PF & $\mathrm{MF}$ & $\mathrm{PF}$ \\
\hline 0 & 0 & 0 & 0 & $0^{-}$ & 0 & 0 & 0 & 0 \\
\hline 4 & 0 & 148 & 0 & 17 & 0 & 2 & 10 & 16 \\
\hline 8 & 0 & 233 & 0 & 87 & 0 & 400 & 0 & 68 \\
\hline 15 & 0 & 12 & 0 & 216 & 0 & 475 & 0 & 116 \\
\hline 22 & 0 & 135 & 0 & 383 & 0 & 383 & 3 & 47 \\
\hline 29 & 0 & 160 & 0 & 188 & 48 & 102 & 2616 & 50 \\
\hline
\end{tabular}

